\title{
Evaluating Switching Overvoltage of a Wind Farm using Monte Carlo Technique \& Fully Digital Parallel Simulators
}

\author{
Jean Belanger ${ }^{1}$, Philippe Venne ${ }^{1}$ and Jean-Nicolas Paquin ${ }^{1}$ \\ ${ }^{1}$ Opal-RT Technologies Inc. \\ 1751 Richardson, Suite 2525 \\ Montreal, Quebec H3K 1G6 Canada \\ Phone/Fax number:+1 514935 2323, e-mail: \{jean.belanger, philippe.venne, jean-nicolas.paquin\}@opal-rt.com
}

\begin{abstract}
The future of the power grid lies in large scale integration of distributed generation devices with the utility system, at either a medium or low voltage level. These new distributed generation technologies can offer benefits and opportunities to manufacturers and utilities in need of supplementary energy sources. However, a large increase in the number of distributed generation interconnections may potentially cause a number of technical concerns relating to the operation of the system in question. Because existing distribution networks were not originally designed to include complex distributed power-electronic systems, detailed testing of existing and future protection and control devices is necessary. The growing use of photovoltaic devices, wind turbines and other complex power electronic systems is changing the nature of distribution systems. The performance and stresses on wind farm components will therefore depend on control and protection system reaction. In fact, this new generation of intelligent grids is becoming as complex as sophisticated high-voltage AC/DC transmission systems. This paper describes how the Monte Carlo simulation technique and parallel simulators can be used to evaluate worst-case stresses for different fault and operating conditions.
\end{abstract}

\section{Keywords}

Real-Time Simulation, Hardware-in-the-Loop, Electromagnetic Transient Simulation (EMT), Wind Turbine models, Fully Digital Real-Time Simulators

\section{Introduction}

Interfacing renewable energy sources (RES) and distributed generation (DG) devices, particularly forced commutation converter-based distributed generation devices, requires meeting strict interconnection requirements from the utility.

Random testing techniques and fast simulators, equipped with replicas of actual control and protection systems, can therefore be used to design and test the performance of intelligent distributed generation and distribution systems against utility requirements, in the same way as they are used for high-voltage AC/DC transmission systems.

Design and test of such complex systems requires evaluation of a wide range of electromagnetic transient phenomena and harmonics that are caused by fast power electronic converters and their controls, and expected during planned manoeuvres and fault conditions. Typical fault sequences involve the operation of multiple breakers and protection \& control systems reacting to system transients. System transients and subsequent protection \& control system operations will in turn be affected by the detailed operation of each subsystem.

Due to the large number of parameters involved, it remains impossible to predict the exact timing sequences of system disturbances that will lead to worst component stresses and controller malfunctions. A larger number of simulation cases involving random variation of key parameters must therefore be performed to find the statistical distribution of overvoltages, overcurrents and arrester energy, as well as protection and control system reaction times and risk of malfunction.

The rapid proliferation of wind farms incorporating fastswitching power electronic devices is therefore posing new challenges for power system engineers responsible for the assessment of global system performance.

This paper begins with a description of the hardware and software architecture of the PC-based Real-Time Power System Simulator used to conduct the electromagnetic transient simulation presented.

A real-time simulation of a wind farm using the Monte Carlo method will be used to evaluate the statistical distribution of overvoltage stresses. Furthermore, this paper will discuss the use of automated random tests to evaluate controller performance and malfunctions in various operating conditions.

Finally, the ability to perform such tests with actual control and protection hardware, connected in closed loop with the simulator, will also be discussed. 


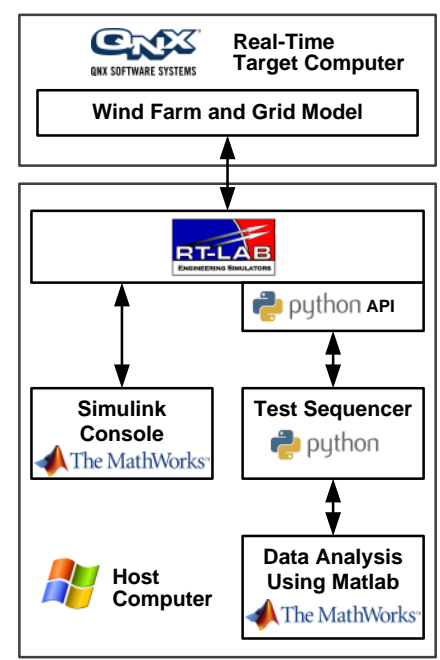

Figure 1: Real-Time Simulator Architecture

\section{Simulator Architecture}

The real-time power system simulator presented in this paper is eMEGAsim from Opal-RT Technologies Inc. eMEGAsim is a distributed, parallel computing real-time simulator designed for the study and simulation of electromagnetic transients (EMT) found in power systems. It enables fast and accurate simulation of EMT phenomena and therefore greater understanding of the power system under study. eMEGAsim is an integrated software and hardware system comprised of Intel multicore processors and FPGA-based I/O. Simulation time steps between 10 to 50 microseconds can be achieved for subsystems executed on the main processors, while models executed on FPGA can achieve time steps below one microsecond.

\section{A. Hardware Architecture}

From a high-level perspective, the experimental setup for the study described in this paper consists of a one 8processor core eMEGAsim simulator. At its core, eMEGAsim has RT-LAB software [1] and Commercialoff-the-Shelf (COTS) components including two Intel ${ }^{\circledR}$ I7 processors, each containing 4 processor cores, and an I/O system based on Xilinx Virtex II TM FPGA processors. eMEGAsim is scalable from 8 to 64 processor cores to simulate increasingly large systems with real-time performance.

Most commercial I/O cards are supported by eMEGAsim to interface the simulator with actual control and protections systems under test. However, a high-density re-programmable FPGA-based I/O system is preferred for electrical HIL applications because such applications require a very large number of very low latency I/O channels. Opal-RT's OP5142 FPGA-based I/O interface card allows for up to 256 channels of time-stamped digital I/O with a resolution as low as 10 nanoseconds, or 128 fast 16-bit analog converters. Multiple FPGA-based I/O subsystems can be connected to the simulator to interface multiple controllers requiring hundreds of I/O channels. Large integration tests of complex VSC converters may require in the range of one to two thousand I/O channels. A very fast communication system based on PCI Express and Direct Memory Access is therefore used to transfer I/O data between the FPGA processors and the main processor to achieve the lowest possible simulation time step.

\section{B. Software Architecture}

eMEGAsim use Red Hat Linux with an optimized kernel as a real-time operating system (RTOS). By so doing, eMEGAsim is able to take advantage of the latest multicore processors and the fastest inter-computer communication systems used in high-performance computer clusters. Maximum communication overhead and jitter can be maintained below 2 to $5 \mu$ s under the worst operating conditions. The use of the open source Linux RTOS on standard computers enables the execution of multiple custom-built and commercially available software applications on the same computer systems used for real-time simulation. This is a clear advantage over the use of custom computers that can execute only one application at a time.

To implement power system models, eMEGAsim relies on tools designed for use with MATLAB/Simulink, a software environment for multidomain simulation and Model-Based Design of dynamic and embedded systems. Simulink has emerged as a worldwide standard for scientific computing and real-time simulation of electromechatronic systems used in aerospace and automotive industries. SimPowerSystems (SPS) extends Simulink ${ }^{\circledR}$ with tools for modeling and simulating the generation, transmission, distribution, and consumption of electrical power. Real-Time Workshop, a C-Code Generator for Simulink, [2] can be adapted to the real-time simulation of power systems when used with ARTEMiS, a solver optimized for real-time simulation of electrical networks [3], and RT-LAB, a real-time distributed software platform. Both ARTEMiS and RT-LAB have been used in a number of industrial sectors for more than 12 years. In addition to SPS/Simulink, ARTEMiS and RT-LAB, eMEGAsim comes equipped with RT-Events and RTeDRIVE; toolboxes especially optimized for real-time simulation of power electronic controls and drive models. These toolboxes have been used for several years by major hybrid vehicle and power electronic system manufacturers [4].

The presented model was built using SPS/Simulink, RT-Events and ARTEMiS [5][6][7].

\section{Automated Test Sequencer Architecture}

The automated test sequencer is a multi-threaded script written in the Python scripting language. The sequencer script acts as a traffic coordinator. It configures the model, synchronises data exchange between the real-time simulator and offline analysis tools and saves and presents simulation results to the user. The sequencer uses as input an Extensible Markup Language (XML) file that specifies the simulation model, all test sequence parameters (constant and random), data acquisition signals, online analysis signals and offline analysis scripts.

The sequencer offers tremendous flexibility in online and offline data analysis. With online analysis, the user can 


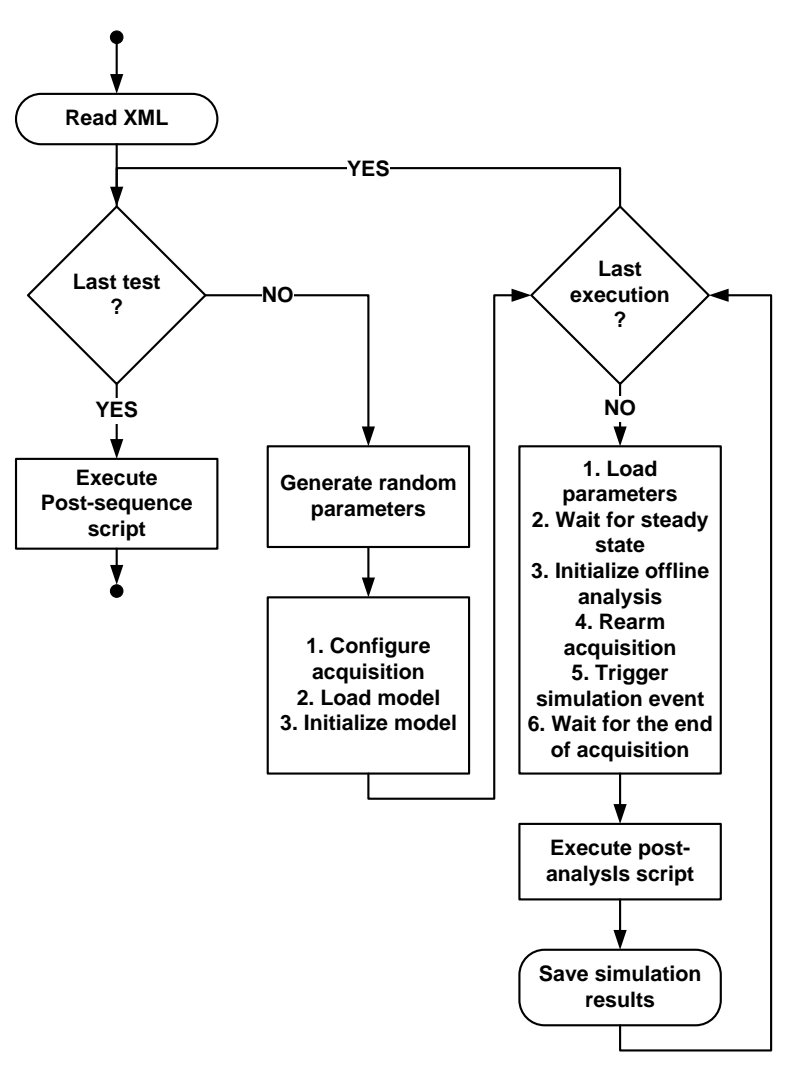

Figure 2: Automated Test Sequencer Execution

insert analysis functions directly in the simulation model using MATLAB/Simulink and retrieve analysis results at the end of each run. Offline analysis offers the possibility to execute complex post-processing of the data while subsequent runs are executed on the real-time simulator. All acquisition and online analysis signals are available for offline analysis, which enables automatic generation of plots and reports.

The execution of the automated test sequencer is illustrated in Figure 2. At the beginning of a sequence, the sequencer reads the XML file, and generates random parameters for each run of the next test. After configuring, loading and initializing the model, the simulation starts. For each simulation run, the sequencer loads the test parameters on the real-time simulator and waits for steady state. It then initializes the offline analysis, rearms the acquisition and triggers the simulation event, which is typically either a fault or circuit breaker switchings. Once the event is triggered, the sequencer waits for the end of data acquisition. It then sends all data to MATLAB for offline analysis and executes the offline analysis script. All data produced is saved. When the last test is completed, a user-defined post-sequence offline analysis script is executed.

\section{Monte Carlo-based Overvoltage Study}

The Monte Carlo simulation technique is commonly used to evaluate switching or low-frequency transient phenomena and control performance during the design of large AC/DC high-voltage transmission systems. Several papers have been published explaining how large analogue simulators, equipped with replicas of actual control and protection systems, take advantage of random techniques to simulate a complete system in real-time in order to evaluate worst case stresses.

Conversely, such simulation of low-frequency electromagnetic phenomena is not needed for insulation coordination of classical low-voltage distribution systems. Equipment design will mainly depend on the evaluation of fast lightning surge overcurrents and overvoltages which are limited by surge arrestors, fuses and other protection systems. Lightning surges and other fast-front overvoltages are typically evaluated by off-line simulation programs, such as EMTP-RV.

However, the integration of Renewable Energy Sources (RES), such as wind farms, to a power grid through distribution lines may create switching and lowfrequency overvoltage that can overstress transformers and other equipment. This section presents how to evaluate such overvoltages using the Monte Carlo technique by programming random sequences of faults and breaker operations and measuring overvoltages computed by the eMEGAsim real-time simulator. Such simulations can be performed in fully numerical mode, where all controlled and protection systems are simulated, or in hard real-time mode, where actual control and protection hardware are connected to the simulator in closed-loop. This is called HIL or Hardware-in-the-Loop simulation, and will be discussed further in Section 4 of the paper.

\section{A. Experiment Description}

Figure 3 presents the power system to be analysed. It is a power system with three different voltage ratings: a High Voltage (HV) section rated at $120 \mathrm{kV}$, a Medium Voltage (MV) section rated at $25 \mathrm{kV}$ and a Low Voltage (LV) section rated at $575 \mathrm{~V}, 60 \mathrm{~Hz}$ with a total generation power of $24 \mathrm{MW}$. Each MV sub-network is connected to a wind farm with a nominal power of $12 \mathrm{MW}$. At each wind farm, the MV section is connected to a capacitor bank to compensate for the reactive power consumed by transmission lines, and to the LV section of each turbine by a three-phase two-winding step-down transformer. The LV section is connected to a Doubly-fed Induction Generator (DFIG) with back-to-back Voltage Source Converter- (VSC) based converters as illustrated on the right side of Figure 3.

The wind turbine model used in the simulation corresponds to the DFIG-based wind turbine model available in the demonstration package of the MATLAB/Simulink SimPowerSystems (SPS) toolbox. The only modification to the SPS model is the replacement of the Universal Bridge block with the 2Level Time Stamped Bridge block included in RTEvents, a library for MATLAB/Simulink, developed by Opal-RT Technologies Inc. A detailed representation of the VSC-based converters is modeled and interfaced to their respective controllers. For fast and real-time simulation, the model has been compiled using RealTime Workshop, a C-Code generator for developing and testing algorithms modeled in MATLAB/Simulink. The model is distributed across 3 processor cores of the eMEGAsim real-time simulator using the RT-LAB real- 

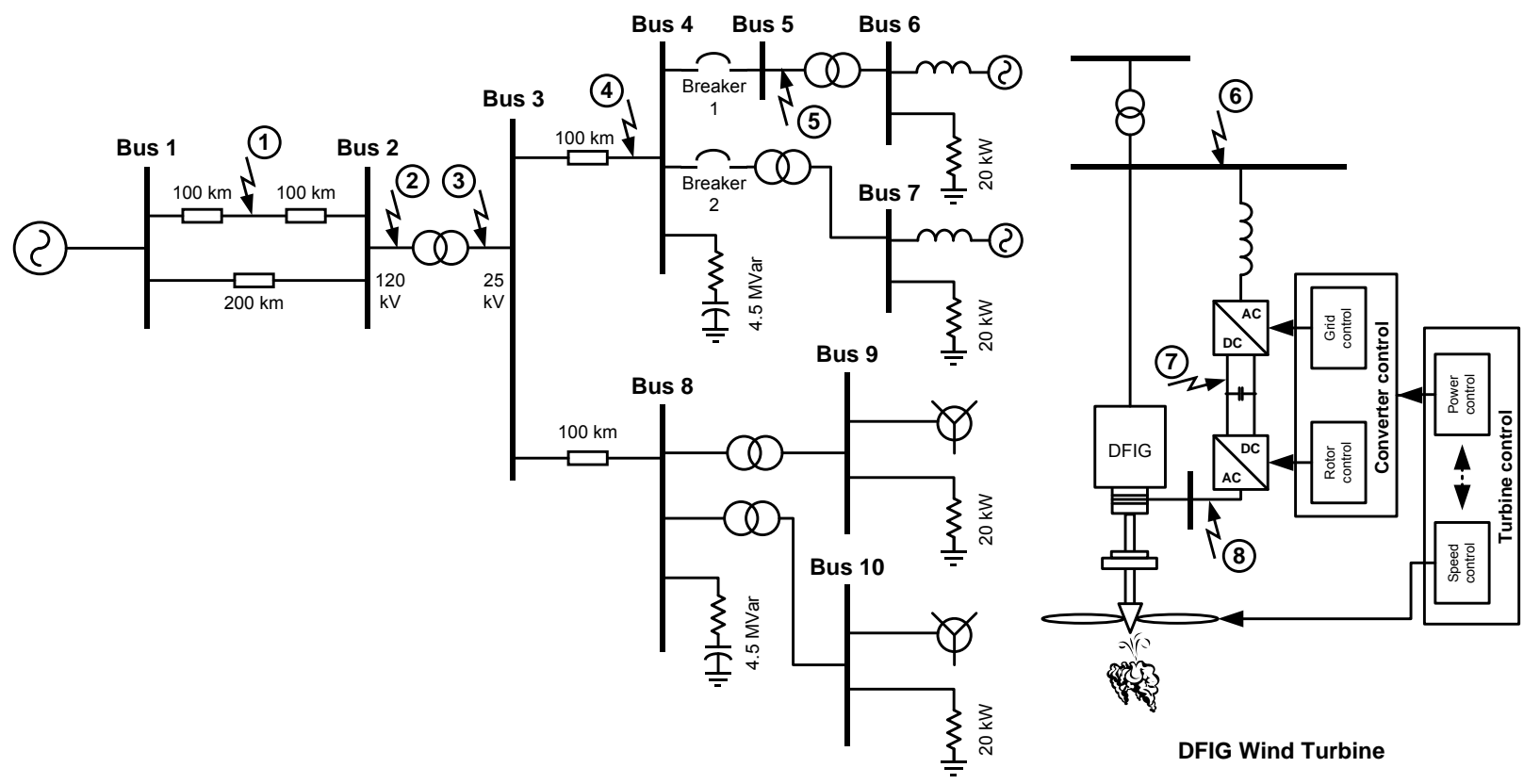

Figure 3: Power System Schematic

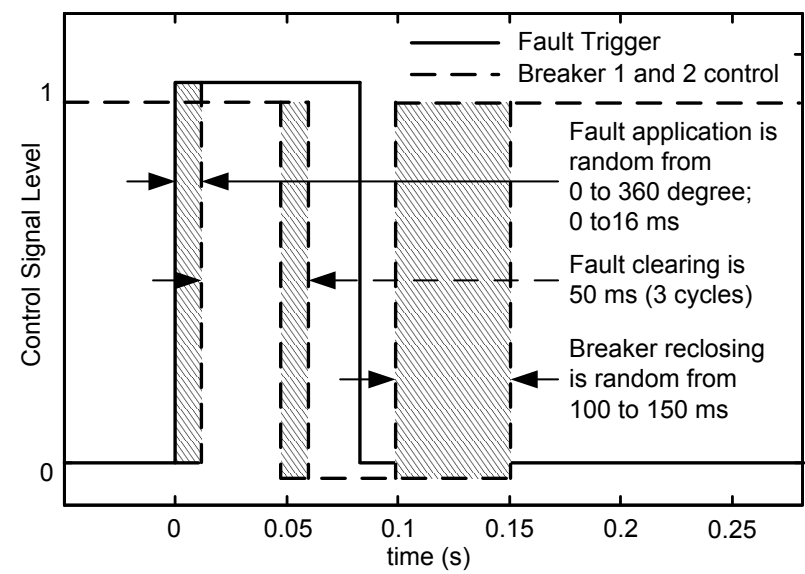

Figure 4: Fault and Breaker Timing during Experiment

time simulation software platform. The discrete simulation time step used was 50 us.

In order to find the stress points of interest, many tests were done on the power system under test. Faults were inserted at 8 different positions. For each position, the 5 fault types outlined in Table 1 were tested. For each test, random point-on wave fault start times from 0 to 360 degrees and random fault durations from 100 to $150 \mathrm{~ms}$ were programmed using a uniform distribution. In this paper, the simulation results are presented for a fault at position 5 followed by the opening of breakers 1 and 2 . This corresponds to a fault that could occur on a wind farm distribution feeder in the field, and the subsequent disconnection of the feeder by a breaker that would clear the fault.

Because of limitations in the wind turbine model, it was not possible to correctly model a fault at the medium voltage bus in front of the wind farm. When this test was executed, the turbine controller would enter an unstable state and never recover after the fault. As a result, for the purpose of this paper, the wind turbine model for the wind farms was kept far from the fault (connected to bus
Table 1: Overvoltage peak value measured on Bus 4 during Faults at Position 5

\begin{tabular}{c|c|c|c|c|c}
\hline Fault Type & $\begin{array}{c}\text { Min } \\
(\mathrm{pu})\end{array}$ & $\begin{array}{c}\mathrm{V}_{2 \%} \\
(\mathrm{pu})\end{array}$ & $\begin{array}{c}\mathrm{Max} \\
(\mathrm{pu})\end{array}$ & $\begin{array}{c}\text { Nb of } \\
\text { runs }\end{array}$ & $\begin{array}{c}\text { Duration } \\
(\mathrm{h})\end{array}$ \\
\hline \hline A-Gnd & 1.93 & 2.95 & 2.96 & 2000 & 4.5 \\
\hline $\mathrm{AB}$ & 1.94 & 3.04 & 3.57 & 2000 & 4.5 \\
\hline $\mathrm{AB}-\mathrm{Gnd}$ & 2.29 & 4.47 & 4.51 & 100 & 0.22 \\
\hline ABC & 2.41 & 3.34 & 3.79 & 100 & 0.22 \\
\hline ABC-Gnd & 2.24 & 3.36 & 3.45 & 100 & 0.22 \\
\hline
\end{tabular}

8 ) and the wind farm placed close to the fault (connected to bus 6 and 7) was modeled with an equivalent ideal source, in this case a reactor. This substitution did not affect the simulation result for voltages measured at bus 4 since the wind farms connected to bus 6 and 7 are disconnected during the fault by the opening of breakers 1 and 2, as illustrated in Figure 4. During the test, once a fault event occurs, the feeder breaker opens $50 \mathrm{~ms}$ later, assuming a full 3 cycle delay for fault detection and breaker opening.

\section{B. Simulation Results}

Table 1 presents simulation results for 5 different fault types. It can be observed that the overvoltage level, having a $2 \%$ or less probability of occurring, commonly referred to as $\mathrm{V}_{2 \%}$, varies from 2.95 to 4.47 per unit across the different fault types. Furthermore, the minimum and maximum overvoltage levels recorded also vary across fault types, reinforcing the argument for running multiple tests with random parameters and fault types in order to identify the overvoltage stress from faults.

In Figure 5 (a) and (c), the overvoltage peak value distribution is illustrated for a phase A to ground and a phase A and B to ground fault. The differences in the shapes of the two distributions and the span between the minimum and the maximum overvoltage levels illustrates the random nature of transient overvoltage amplitude following the application and clearing of a fault, and the 


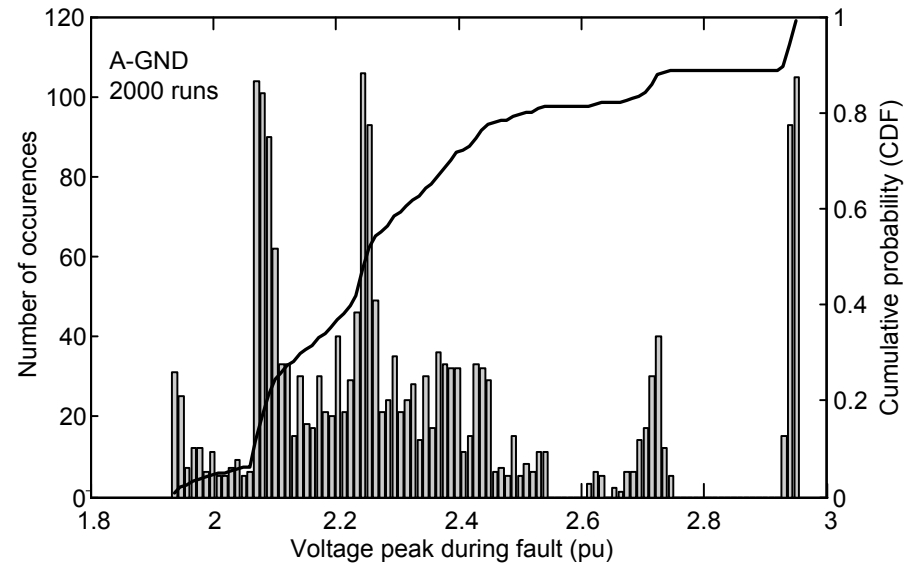

(a)

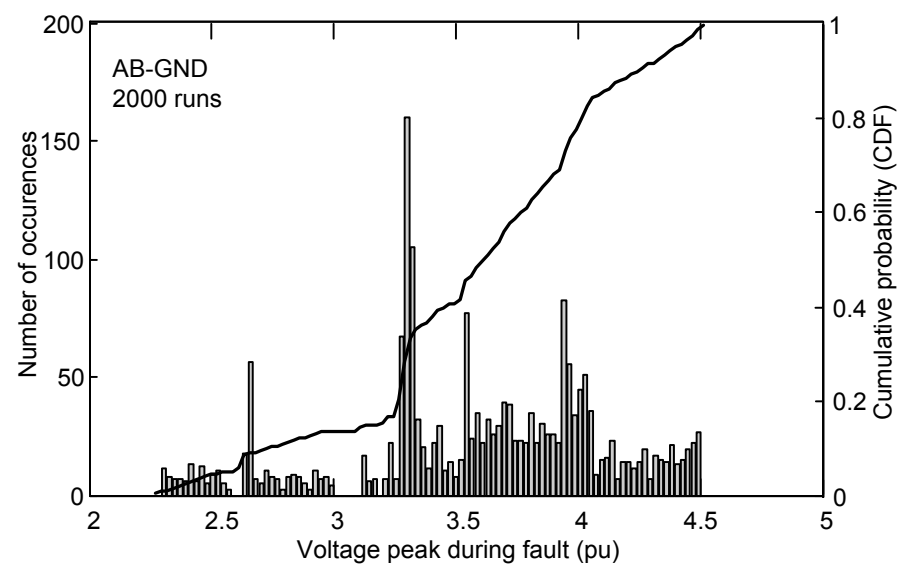

(c)

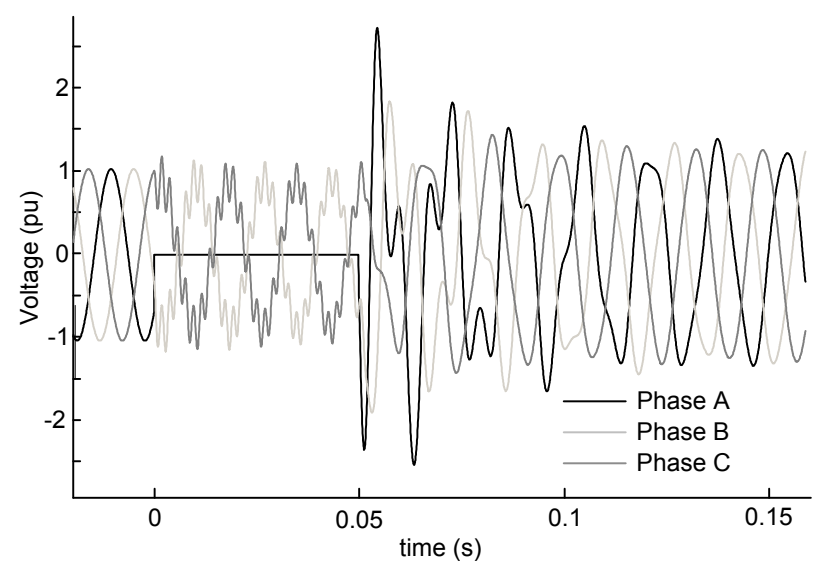

(b)

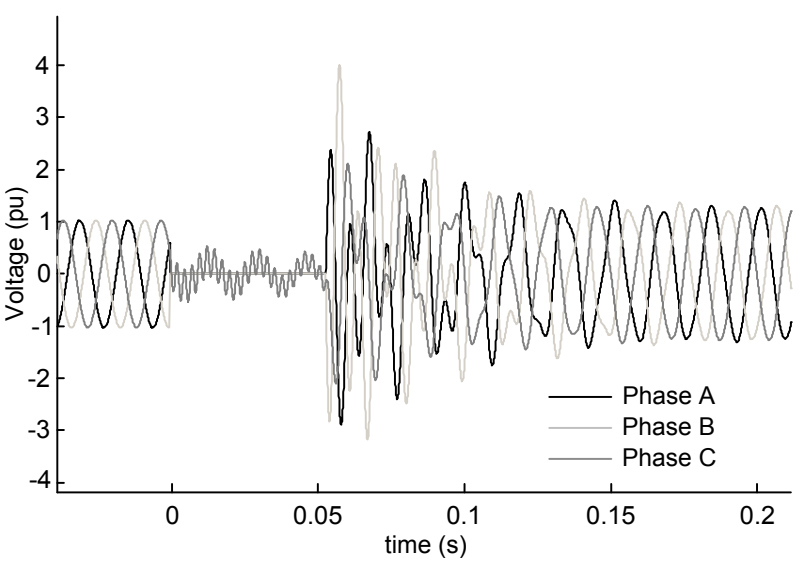

(d)

(a) Overvoltage peak value distribution at Bus 4 for an A to Ground fault; (b) Sample Voltage at Bus 4 for an A to Ground fault; (c) Overvoltage peak value distribution at Bus 4 for an AB to Ground fault; (d) Sample Voltage at Bus 4 for an AB to Ground fault; Figure 5: Monte Carlo Simulation Results for Faults at Position 5

necessity to run a large number of tests to quantify the $\mathrm{V}_{2 \%}$ level related to a specific fault type and position.

Figure 5 (b) and (d) present sample voltage waveforms for a phase A to ground and a phase A and B to ground fault. From these waveforms it can be observed that worst-case overvoltage stress occurs not at the beginning of the fault but after the disconnection of the feeder line of the wind farm, when breakers 1 and 2 are opened.

To evaluate the relationship between the number of test runs based on random parameters and the $\mathrm{V}_{2 \%}$ level found for a specific fault type and location, the $\mathrm{V}_{2 \%}$ level was computed for every added test run from the $100^{\text {th }}$ test to the last test of a test sequence of 2000 runs. It can be observed from Figure 6 that the $\mathrm{V}_{2 \%}$ level varies during the first few hundred test runs, and then stabilizes around the 900th test run. It can therefore be concluded that for this specific test, about 900 tests would be necessary to properly evaluate the $\mathrm{V}_{2 \%}$ level, depending on the level of accuracy specified for the analysis.

\section{Discussion}

The simulation results presented illustrate the random nature of voltage peak values occurring at fault clearing in wind farms. From these results, the use of real-time simulation tools, combined with Monte Carlo methods, can be proposed as an efficient technique to evaluate overvoltage stresses in wind farms.

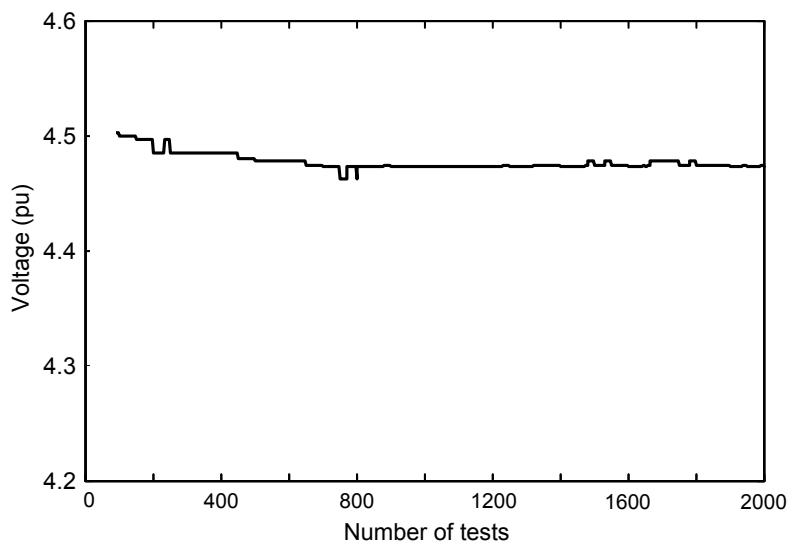

Figure 6: Overvoltage peak value on Bus 4 at $2 \%$ probability as a function of the number of test runs for an A to Ground Faults at Position 5

One of the advantages of real-time over offline simulation when using Monte Carlo methods is the reduction of testing time and the possible increase in test coverage that comes from the ability to increase the number of tests as the testing time gets smaller. The time required to complete each test varies from 18 minutes to 4.5 hours, depending on the number of random fault applied for the tests. Such duration can be further reduced by reducing the time to stabilize the system between each run, or by setting the initial condition. 


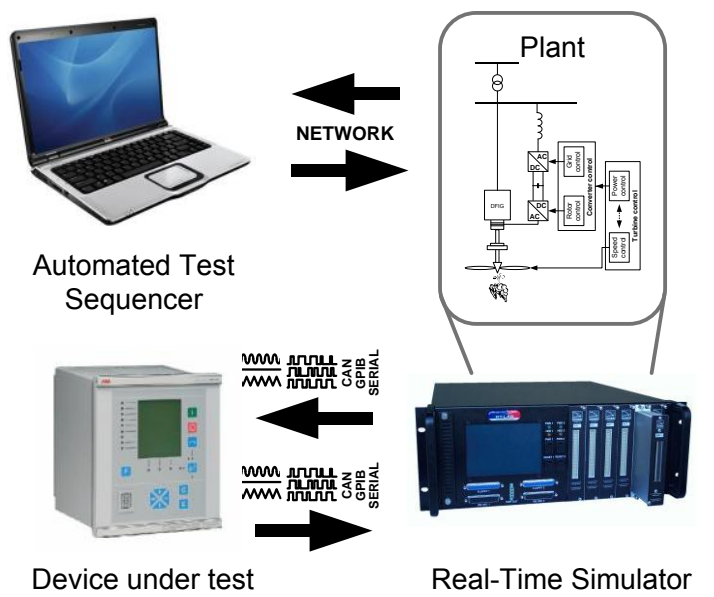

Figure 7: HIL Simulation

However, conducting such tests with standard single processor software will not be practical for large power grids equipped with a large number of powe electronic systems.

Furthermore, random parameter variation could be used to trigger any type of event in the model while it is executed on the real-time simulator. This functionality could therefore be used to test the robustness of control algorithms to defective sensors and actuators, to unusual operating conditions, or to any other event.

\section{Hardware-in-the-Loop (HIL)}

There are times when physical pieces of equipment must be part of the software simulation of a system. This can be the case when the study objective is to test a hardware prototype or final control \& protection systems. In such cases, the HIL simulation method is used, where physical hardware is connected to the real-time simulator by analog and digital inputs \& outputs. The integration of physical hardware devices in a real-time simulation offers many advantages. By testing a device as an integrated physical system, its true functionality can be determined including all its interfaces. And with the help of a test sequencer, a large number of repetitive and random tests can be run on the device, uncovering conditions that could not have been discovered otherwise. Furthermore, tests that would have been too risky or expensive to perform on a real power system become possible.

With the help of real-time simulation, interactions between a new device and other control \& protection systems, whether they are simulated or implemented in final hardware connected via HIL, can be easily analyzed and tested for a variety of normal and fault conditions. Therefore, when analytical methods fail to provide rigorous data on power system stability margins and currents and voltage intensity limits, statistical methods such as Monte Carlo studies, combined with real-time simulation and HIL testing, can help supply the missing data needed for properly defining specifications of power system components [8].

\section{Conclusion}

This paper presented a description of the hardware and software architecture of a PC-based Real-Time Power System Simulator used to conduct electromagnetic transient simulations. Applying the simulator to the identification of the statistical distribution of overvoltage stresses in a wind farm was also presented. The ability to perform such tests with actual control and protection hardware, connected in closed-loop with the simulator, was also discussed.

With the increased complexity of power systems and the introduction of VSC-based renewable energy sources, real-time simulators combined with automated test sequencers will allow the evaluation, not only of stresses, but also controller stability margins, sensor error tolerances and many other parameters useful for the optimal operation of power systems.

\section{References}

[1] L.-F. Pak, M. O. Faruque, X. Nie, V. Dinavahi, "A Versatile Cluster-Based Real-Time Digital Simulator for Power Engineering Research," IEEE Trans. Power Systems, vol. 21, no. 2, pp. 455-465, May. 2006.

[2] H. Jones, "Return on Investment in Simulink for Electronic System Design", White Paper [Online]. Available:

http://www.mathworks.com/mason/tag/proxy.html?dataid $=5872 \&$ fileid $=24121$

[3] C. Dufour, J. Bélanger, "Discrete Time Compensation of Switching Events for Accurate Real-Time Simulation of Power Systems", in Proc. 2001 IEEE Industrial Electronics Society Conference, pp. 1533-1538.

[4] M. Harakawa, H. Yamasaki, T. Nagano, S. Abourida, C. Dufour and J. Bélanger, "Real-Time Simulation of a Complete PMSM Drive at 10 us Time Step," Proceedings of the 2005 International Power Electronics Conference (IPEC 2005), Niigata, Japan, April 4-8, 2005.

[5] C. Dufour, J. Bélanger, "Real-time Simulation of a 48Pulse GTO STATCOM Compensated Power System on a Dual-Xeon PC using RT-LAB," Proceedings of the International Conference on Power Systems Transients (IPST 2005), Montréal, Canada, June 19-23, 2005

[6] C. Dufour, J. Bélanger, "Discrete Time Compensation of Switching Events for Accurate Real-Time Simulation of Power Systems", in Proc. 2001 IEEE Industrial Electronics Society Conference, pp. 1533-1538.

[7] J. Bélanger, L. A. Snider, J. N. Paquin, C. Pirolli, W. Li, "A Modern and Open Real-Time Digital Simulator of Contemporary Power Systems", Proceedings of the International Conference on Power Systems Transients (IPST 2009), Kyoto, Japan, 2-6 June, 2009.

[8] J. .-N. Paquin, J. Bélanger, L. A. Snider, C. Pirolli, and W. $\mathrm{Li}$, "Monte-Carlo Study on a Large-Scale Power System Model in Real-Time using eMEGAsim," in , San-José, 2009.

[9] C. Larose, R. Gagnon, G. Turmel, P. Giroux, J. Brochu, D. McNabb, D. Lefebvre, "Large Wind Power Plants Modeling Techniques for Power System Simulation Studies" presented at 8th International Workshop on Large-Scale Integration of Wind Power into Power Systems - Bremen, Germany, 14-15 October 2009 